\title{
Quantum radiation reaction in aligned crystals beyond the local constant field approximation
}

\author{
T. N. Wistisen $\odot,{ }^{2,1}$ A. Di Piazza, ${ }^{2}$ C. F. Nielsen $\odot,{ }^{1}$ A. H. Sørensen, ${ }^{1}$ and U. I. Uggerhøj $\oplus^{1}$ \\ (CERN NA63) \\ ${ }^{1}$ Department of Physics and Astronomy, Aarhus University, 8000 Aarhus, Denmark \\ ${ }^{2}$ Max-Planck-Institut für Kernphysik, Saupfercheckweg 1, D-69117, Germany
}

(Received 21 June 2019; published 9 October 2019)

\begin{abstract}
We report on experimental spectra of photons radiated by $50-\mathrm{GeV}$ positrons crossing silicon single crystals of thicknesses 1.1, 2.0, 4.2, and $6.2 \mathrm{~mm}$ at sufficiently small angles to the (110) planes that their motion effectively is governed by the continuum crystal potential. The experiment covers a regime of interaction where each positron emits several hard photons, whose recoils are not negligible and which are formed on lengths where the variation of the crystal field cannot be ignored. As a result, neither the single-photon semiclassical theory of Baier et al. nor the conventional cascade approach to multiple hard photon emissions (quantum radiation reaction) based on the local constant field approximation are able to reproduce the experimental results. After developing a theoretical scheme which incorporates the essential physical features of the experiments, i.e., multiple emissions, photon recoil, and background field variation within the radiation formation length, we show that it provides results in convincing agreement with the data.
\end{abstract}

DOI: 10.1103/PhysRevResearch.1.033014

\section{INTRODUCTION}

Strong electromagnetic fields such as those produced by intense lasers and by crystals are a unique tool to test QED in the laboratory in unprecedented high-energy regimes, where nonlinear effects in the electromagnetic field energy density dominate the dynamics [1-6]. When electrodynamical processes occur in the presence of a sufficiently intense background electromagnetic field, the photon density of the latter is so high that charged particles like positrons (charge $e$ and mass $m$, respectively) interact coherently with several background field photons. The theoretical description of this regime, known as strong-field QED (SFQED), relies on Lorentz- and gauge-invariant parameters, which depend on the structure of the external electromagnetic field [7].

When a high-energy positron impinges onto a crystal along a symmetry plane of the crystal lattice, its motion can become transversely bound between two adjacent planes, and the positron effectively interacts with a static "continuum" potential varying only along the direction perpendicular to the planes (planar channeling) [3,5,8-10]. In planar channeling, the transverse motion decouples from the motion along the $y-z$ plane and the condition for a positron with initial energy $\varepsilon_{0} \gg m$ to be channeled within two planes is that the total kinetic plus potential energy $U(x)$, such that $\varepsilon_{x}=p_{x}^{2}(t) / 2 \varepsilon_{0}+$ $U(x(t))$ associated to the transverse motion is smaller than the potential energy height $U_{0}$ between the two planes [3,5,8-10]. Here, we have assumed that $x$ is the coordinate perpendicular to the symmetry planes and that $p_{x} \ll \varepsilon_{0}$ is the positron

Published by the American Physical Society under the terms of the Creative Commons Attribution 4.0 International license. Further distribution of this work must maintain attribution to the author(s) and the published article's title, journal citation, and DOI. momentum along that direction (units with $\hbar=c=1, \alpha=e^{2}$ are employed). The condition for planar channeling can be expressed as a bound on the maximal positron angle $\theta$ to the plane while in the crystal that has to be smaller than $\theta_{c} \equiv \sqrt{2 U_{0} / \varepsilon_{0}}$ [3]. The continuum approximation also applies for $\theta \gtrsim \theta_{c}$ if $\varepsilon_{0} \gg m$.

The study of SFQED processes in the background crystal field corresponding to the continuum potential, such as the emission of high-energy photons, is complicated by the necessity of including the field exactly in the calculations. Now, in the case of planar channeling, the crystal field has a dependence on the coordinate $x$, which does not allow for an exact analytical solution of the Dirac equation [11]. For this reason, the semiclassical method of Baier and Katkov [3,12], which allows for the computation of the probabilities of quantum processes using only the classical trajectory of the charged particles involved in the process, has been extremely useful in the study of SFQED processes. The semiclassical method is based on the observation that in the interaction of ultrarelativistic particles (we consider positrons here) the quantization of the motion is negligible such that one can still attribute physical meaning to the positron classical trajectory, whereas the main quantum effect in the process of radiation to be included is the recoil in the emission of high-energy photons [3]. The semiclassical method has been successfully employed to compute the probability of the basic SFQED processes like single photon emission and electron-positron photoproduction in aligned crystals (see Ref. [3] also for studies on higher order processes). However, when a positron crosses a crystal whose thickness corresponds to several radiation lengths, a potentially large number of photons can be emitted. The theoretical investigation of such high-order processes is a formidable task [13] and mainly kinetic approaches are employed, where it is assumed that multiple-photon emissions arise from sequential (cascade) emissions of single photons, 
with each single-photon emission being well localized [3]. The localization of the emission is a crucial requirement of the method and it corresponds to assuming that the formation length $l_{f}$ of the photon emission process is much smaller than the typical length where the crystal field significantly varies, such that the local value of the probability per unit time, evaluated for a constant field, can be employed [2,3]. This local constant field approximation (LCFA) is another remarkable tool in strong-field physics and recent studies have been devoted to investigating its limitations, especially in the realm of SFQED in beamstrahlung $[14,15]$, in intense laser fields [16-19], and in space-time dependent electric fields [20]. The LCFA has previously been applied to highenergy radiation and pair-production processes in aligned single crystals. In Refs. [21,22], the leading-order correction in the field derivatives of the photon radiation probability has been found. In Refs. [23,24], experimental results beyond the LCFA are presented but either quantum radiation reaction effects were negligible, i.e., each charge emits on average one photon or, in case of multiple-photon emission, singlephoton spectra were not measured. See Refs. [25-28] for other channeling related effects.

Here, we report experimental single-photon spectra emitted by high-energy $(50-\mathrm{GeV})$ positrons crossing silicon crystals of different thicknesses $(1.1,2.0,4.2$, and $6.2 \mathrm{~mm})$ aligned to the (110) planes. Depending on the crystal thickness, several photons are emitted by each positron with significant recoil, such that quantum radiation reaction effects have to be taken into account (see also the results of our previous experiment reported in Ref. [29]). By employing a conventional kinetic approach based on the emission probabilities evaluated within the LCFA, we show that such an approach is unable to explain the experimental results. Thus, we have developed a kinetic approach suitable for SFQED processes in crystals oriented along crystallographic planes, but which can, barring extreme circumstances, be adapted to any external field for which the transverse velocity of the particle, as obtained from the Lorentz-force equation, is (approximately) periodic, which then includes effects beyond the LCFA; see the Appendix. The extreme circumstances refer here to the fact that the motion must remain semiclassical; see, e.g., Ref. [30] on this topic. Other examples of such a field include those of a long laser pulse, of long dense particle bunches (as in beamstrahlung), and of undulators or wigglers. The theoretical spectra obtained with this method result in overall good agreement with the data, which in turn can be interpreted as experimental evidence of quantum radiation reaction beyond the LCFA.

\section{EXPERIMENT}

The experiment was carried out at the CERN SPS H4 beamline employing a positron beam of $50 \mathrm{GeV}$ with an energy spread of a few percent (see Fig. 1). Because of changed conditions of the accelerator, which can occur during outages, the positron beam features varying initial angular distributions along the $x$ direction (the crystal symmetry planes are defined to be parallel to the $y-z$ planes). The experimental angular distributions were fitted with a Gaussian function and the resulting average angle $\theta_{0}$ relative to the crystal plane and

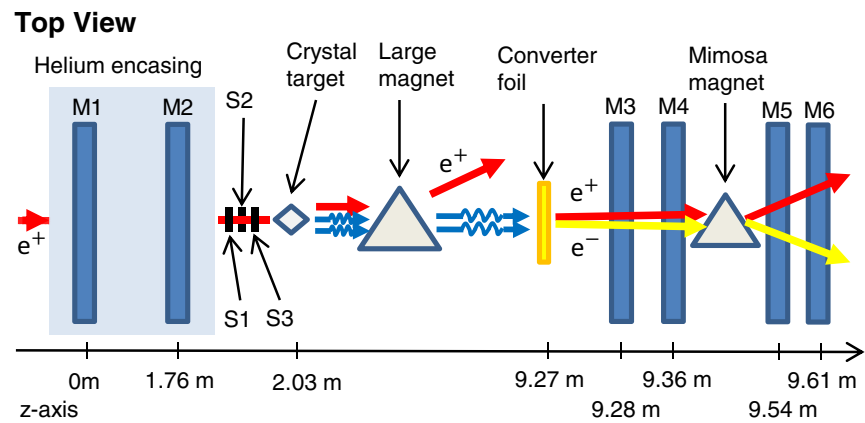

FIG. 1. A top view schematic of the experimental setup.

standard deviation $\sigma_{\theta}$ are reported in Table I for the crystal thicknesses used in the experiment. As the radiation spectra are highly sensitive to the entry angle of the positrons, the variation in the entry angles complicates a direct comparison between spectra for the different crystal thicknesses.

The scintillators $\mathrm{S} 1, \mathrm{~S} 2$, and $\mathrm{S} 3$ are used to make the trigger signal, for which a signal must be present in $\mathrm{S} 1$ and $\mathrm{S} 3$ and absent in S2, as S2 has a hole to allow particles through. After the scintillators, the positron enters a helium chamber to reduce multiple Coulomb scattering. Here, the transverse position of the positron is measured as it passes through the MIMOSA detectors M1 and M2, which allows us to determine the incidence angle. The positron then enters the silicon crystal, where it emits radiation. A large magnet removes the charged particles exiting the crystal. The emitted photons instead continue forward and encounter a converter foil of $200-\mu \mathrm{m}$ tantalum, corresponding to an approximately $1 / 26$ chance of being converted into an electron-positron pair. Note that when the photon energy exceeds the threshold of pair production, the pair production cross section quickly tends to a constant value for large photon energies. This approach of conversion is used to obtain a spectrum of individual photons, as opposed to a calorimeter setup, which would only measure the sum of energies of all the emitted photons. The produced electron and positron pair is tracked through detectors M3 and M4, then deflected by another magnet, and then tracked again in M5 and M6. The deflection angles of the electron and positron allow us to determine their individual momenta, whose sum yields the momentum of the original photon (see Ref. [29] for a description of the employed tracking algorithm). The response of the experimental setup is complicated

TABLE I. Average angles $\theta_{0}$ and standard deviations $\sigma_{\theta}$ of the Gaussian functions fitting the initial angular distribution of the positrons along the $x$ direction for the various crystal thicknesses used in the experiment. For the case of our experiment, we have $\theta_{c}=30 \mu \mathrm{rad}$; that is, only a minor fraction of positrons is channeled. Note that the critical angle $\psi_{p}$, as given, e.g., in Ref. [5], assumes the value $\psi_{p}=23 \mu \mathrm{rad}$.

\begin{tabular}{lccccc}
\hline \hline Case & $\mathrm{a}$ & $\mathrm{b}$ & $\mathrm{c}$ & $\mathrm{d}$ & $\mathrm{e}$ \\
\hline$L[\mathrm{~mm}]$ & 6.2 & 2.0 & 6.2 & 4.2 & 1.1 \\
$\sigma_{\theta}[\mu \mathrm{rad}]$ & 100 & 100 & 85 & 85 & 85 \\
$\theta_{0}[\mu \mathrm{rad}]$ & 50 & 70 & 7 & 62 & 27 \\
\hline \hline
\end{tabular}


by multiple scattering through the setup, finite detector sizes, etc., and therefore it should be simulated [29]. In order to validate the simulation of the experiment, the crystal can be oriented far away from any low-index crystallographic direction, such that the emission of radiation essentially stems from Bethe-Heitler $(\mathrm{BH})$ bremsstrahlung rather than showing coherence effects as in coherent bremsstrahlung or channeling radiation. $\mathrm{BH}$ bremsstrahlung is a well-studied process, and the agreement between the simulation using the $\mathrm{BH}$ spectrum and the experimental spectra shows that the experimental setup is well understood and described by the simulation. An overall normalization constant is used on the simulation such that the $\mathrm{BH}$ simulation matches the experiment, and this accounts for the inherent efficiency in the MIMOSA detectors.

\section{THEORY}

For the theoretical description of the experimental results, it is useful to introduce the parameters $\chi=e\langle\varepsilon E\rangle / \mathrm{m}^{3}$ and $\xi=$ $\left|\boldsymbol{p}_{\perp, \max }-\left\langle\boldsymbol{p}_{\perp}\right\rangle\right| / m$ [1-5]. Here, $\varepsilon(t)$ is the positron energy at time $t, E(t)$ is the amplitude of the crystal electric field at the positron position at time $t$, the symbol \langle\rangle indicates the average over the positron trajectory, and $\left|\boldsymbol{p}_{\perp, \max }\right|$ is the maximum momentum transverse to the direction of the largest component of the momentum $p_{z}(t) \approx \varepsilon(t)$ (note that for channeled positrons $\left\langle p_{x}\right\rangle=0$ ). When $\chi$ is of the order of unity or larger, quantum effects such as spin and recoil during the emission are essential. The parameter $\xi$ differentiates between regimes of undulator-like $(\xi \ll 1)$ and synchrotron-like $(\xi \gg 1)$ radiation emission. Quantum radiation reaction is the emission of multiple photons while quantum effects in the emission is important, i.e., $\chi$ is not too small [31]. When $\xi \gg 1$, the calculation of the quantum radiation reaction process is simplified as one can assume that the multiple emissions mainly stem from a sequence of localized single-photon events and use the LCFA to calculate the corresponding single-photon radiation emission probability. This approach has been employed to explain recent experimental results on radiation reaction $[29,32,33]$. When $\xi$ is on the order of unity, the above approach is no longer applicable, and a more general theory of radiation reaction is required. In the experiment reported here, we have that $\xi<2.9$ and $\chi<0.042$, with the inequalities being due to different initial conditions of the positron yielding different values of the parameters. While the value of $\chi$ is smaller than unity, it is large enough that quantum effects are important in the experiment. This important point is illustrated in Fig. 2 by a direct comparison of classical and quantum spectra of radiation emission (average energy radiated per unit of photon energy and unit length) for a thin crystal, such that radiation reaction effects, i.e., multiple photon emission, can be neglected.

Since quantum effects are important, we compare the experimental data with three theoretical quantum models. In the first and most general model, called quantum stochastic model (QSM), the multiple-photon emission is treated as a cascade of sequential single-photon emissions. Each single-photon emission event is implemented via a Monte Carlo approach based on probabilities which are summed over the final spin and photon polarization, and averaged over the initial spin. The important feature of this model is the use of the semiclas-

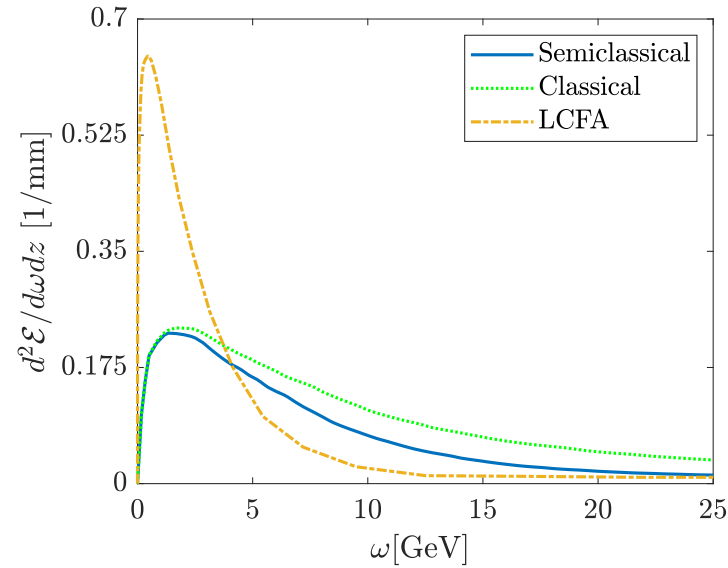

FIG. 2. Theoretical single-photon power spectra for a positron beam of $50 \mathrm{GeV}$ energy crossing a crystal of $0.1 \mathrm{~mm}$ thickness, which is the energy emitted per unit photon energy and per unit length of the crystal. For the incoming positron angular distribution, we have chosen $\theta_{0}=7 \mu \mathrm{rad}$ and $\sigma_{\theta}=85 \mu \mathrm{rad}$. The green, dotted curve corresponds to the classical emission spectrum; the blue, continuous curve corresponds to the quantum emission spectrum via the semiclassical method; and the yellow, dashed curve corresponds to the quantum emission spectrum within the LCFA. See also Fig. 1 in Ref. [22].

sical method of Baier and Katkov to calculate the differential single-photon emission rate $d W / d \omega[3,15,34-37]$ integrated over a finite section of the positron trajectory corresponding to a finite time interval $T$. This approach is then able to handle quantum radiation reaction beyond the LCFA for planar channeling and takes advantage of the regular, oscillatory motion of positrons inside the crystal field. In fact, the value of $T$ has to be large enough such that the differential rate $d W / d \omega$ has converged, i.e., it no longer changes significantly when $T$ is further increased. This requires a value of $T$ on the order of several photon formation lengths $l_{f}=2 \gamma^{2}(1-\omega / \varepsilon) / \omega[3]$ or several wavelengths of the particle motion $\lambda$, whichever is larger, where $\gamma=\varepsilon / m$ is the Lorentz factor of the positron at the moment of emission. We refer to the Appendix for more details on this scheme.

The second model is the LCFA, which is the usual approach to quantum radiation reaction, where multiple-photon emissions are simulated via independent and random emission events, with the emission probability being used within the LCFA [29]. Finally, the third model is the "no RR," where radiation reaction is ignored, which is the same as the first model, except that the momentum of the emitted photon is not subtracted from the radiating positron. The difference between the first and the third models, therefore, shows the size of radiation-reaction effects. In Ref. [29], we described how to use the constant field approximation in the case of channeling radiation and therefore we refer to this paper for additional details. The only difference in the LCFA model as compared to that employed in Ref. [29] is that here we also add the incoherent $\mathrm{BH}$ bremsstrahlung with a Monte Carlo approach. The reason is that this process is more important here than in Ref. [29].

The process of multiple elastic scattering of the positron with the nuclei as the positron propagates through the crystal 

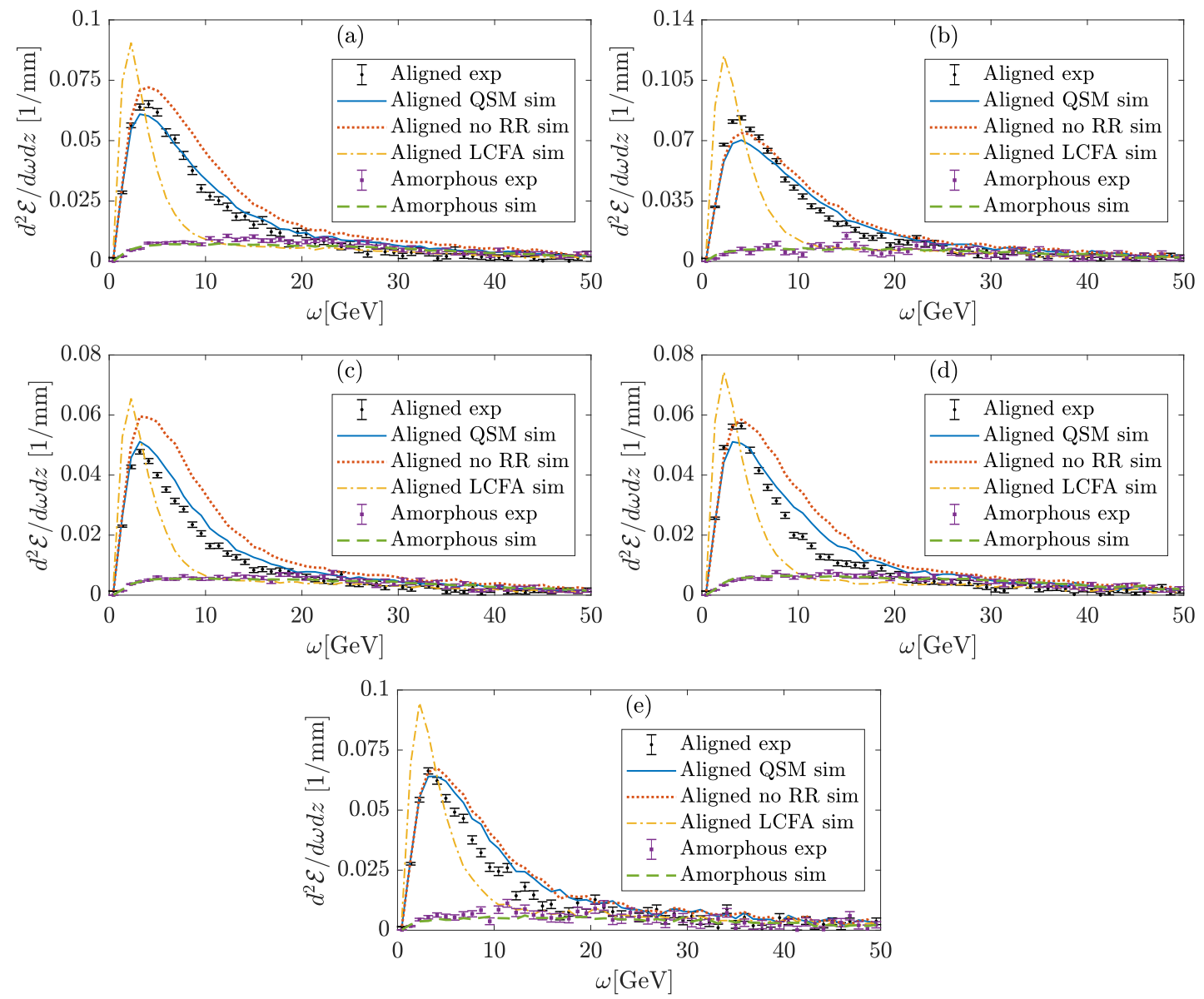

FIG. 3. Experimental data compared with the three different models described in the text in the "aligned" case. The data with the crystal turned into the "amorphous" orientation is compared with the simulation of the BH bremsstrahlung spectrum. See Table I for the parameters corresponding to each subplot.

plays an important role as this on average increases the otherwise conserved energy $\varepsilon_{x}=p_{x}^{2}(t) / 2 \varepsilon_{0}+U(x(t))$ associated with the motion along the $x$ direction. Since the radiation emission spectrum depends on this effect, we have implemented it in our numerical codes as described in Ref. [38]. The positron velocity at each time step, as provided by the solver of the trajectory according to the Lorentz force, is additionally changed by an amount which is random and normal distributed, with a standard deviation depending on the local density of nuclei and electrons. In Ref. [39], a method similar to what we have described here was put forward. However, the method was compared to experimental results with $\xi \gg 1$, where the LCFA was a good approximation. Moreover, an important difference between the two methods is that in Ref. [39] the trajectory is divided into sections with length of the order of the period of motion which in general does not lead to convergence of the differential rate (this is, however, acceptable at $\xi \gg 1$, for which it was applied, because in this case the formation length is generally shorter than the oscillation period).

\section{DISCUSSION OF RESULTS}

In Fig. 3, we show all the experimental data corresponding to five different settings of crystal thickness and beam distributions; see Table I. In all figures, we also report the simulation corresponding to the amorphous orientation and we always find a very good agreement with the $\mathrm{BH}$ bremsstrahlung, which is a good validation of the simulation of the experimental setup. Additionally, Fig. 3 shows that the three models give three distinct curves, which means that we are able to distinguish between the two models of radiation reaction (within and beyond the LCFA) as well as to establish that radiation reaction is present, as otherwise the "no-RR" curve would coincide with the QSM curve. The no-RR curve is naturally higher than the QSM curve because at given conditions the radiation power of a charge increases for higher charge energies, and the inclusion of radiation reaction lowers the energy of the positron as it propagates through the crystal. One may note that the relative height of the LCFA curve in the theory plots from Fig. 2, compared to the other models, is larger than the corresponding curves seen when comparing with the experimental data in Fig. 3. This is because the simulation of the experimental setup takes into account that the latter has a lower detection efficiency for low photon energies. The primary reason for this is that the pairs produced by a low-energy photon will scatter so much in the converter foil that one of or both the produced particles have an angle too large to hit within the area of the MIMOSA detector. In Fig. 3, it is, however, clearly seen that 
the LCFA is not applicable in the parameter regime under investigation. Indeed, the LCFA approximation neglects interferences between the contributions to the radiation emission amplitude between different parts of the trajectory. Instead, the stochastic approach presented here takes these effects into account by performing the integration over several photon formation lengths and particle oscillations along the positron trajectory. Figure 3 indeed shows that the QSM model is overall in good agreement with the experimental data.

\section{CONCLUSION}

In conclusion, we have presented experimental results in a regime of quantum radiation emission where available theoretical models based on the local constant field approximation are inadequate. By putting forward another approach to the quantum radiation reaction, which takes into account effects beyond the local constant field approximation, we have obtained results in convincing agreement with the experimental data.

\section{ACKNOWLEDGMENTS}

T. Wistisen was supported by the Alexander von Humboldt-Stiftung except for the initial part of the project, where he was supported by the VILLUM FONDEN (Research Grant No. VKR023371). Except for the first author, the author list is alphabetical and the contribution of each author was the following: T. N. Wistisen and U. I. Uggerhøj conceived and designed the experiment. C. F. Nielsen carried out the data analysis with assistance from U. I. Uggerhøj and A. H. Sørensen. C. F. Nielsen, U. I. Uggerhøj, A. H. Sørensen, and T. N. Wistisen participated in the experiment. T. N. Wistisen proposed the theoretical models in collaboration with A. Di Piazza and carried out the numerical calculations. T. N. Wistisen and A. Di Piazza wrote the paper with input from the rest of the collaboration and C. F. Nielsen produced the figures with the experimental data.

\section{APPENDIX}

If the Lorentz-force motion of the particle has a velocity that is approximately transversely periodic, one can write the differential emission rate per photon solid angle $\Omega$ and energy $\omega$ by a positron as $[3,35,37]$

$$
\frac{d^{2} W}{d \omega d \Omega}=\lim _{T \rightarrow \infty} \frac{1}{T} \frac{1}{\omega} \frac{e^{2}}{4 \pi^{2}}\left(\frac{\varepsilon^{2}+\varepsilon^{2}}{2 \varepsilon^{2}}\left|\boldsymbol{I}_{T}\right|^{2}+\frac{\omega^{2} m^{2}}{2 \varepsilon^{4}}\left|J_{T}\right|^{2}\right),
$$

where $\varepsilon$ is the initial positron energy, $\varepsilon^{\prime}=\varepsilon-\omega$, and

$$
\begin{gathered}
\boldsymbol{I}_{T}=\int_{-T / 2}^{T / 2} \frac{\boldsymbol{n} \times[(\boldsymbol{n}-\boldsymbol{v}) \times \dot{\boldsymbol{v}}]}{(1-\boldsymbol{n} \cdot \boldsymbol{v})^{2}} e^{i k^{\prime} x} d t, \\
J_{T}=\int_{-T / 2}^{T / 2} \frac{\boldsymbol{n} \cdot \dot{\boldsymbol{v}}}{(1-\boldsymbol{n} \cdot \boldsymbol{v})^{2}} e^{i k^{\prime} x} d t,
\end{gathered}
$$

with $k^{\prime \mu}=\left(\varepsilon / \varepsilon^{\prime}\right) k^{\mu}, k^{\mu}=(\omega, \boldsymbol{k})=\omega(1, \sin \theta \cos \varphi, \sin \theta \sin \varphi$, $\cos \theta), \boldsymbol{n}=\boldsymbol{k} / \omega$, and with $\boldsymbol{v}(t)$ and $\dot{\boldsymbol{v}}(t)$ being the positron velocity and the acceleration at time $t$ as obtained by solving the Lorentz-force equation of motion. Usually the formulas are given in terms of emission probability, and the ratio of the probability with the integration length $T$ converges, yielding a rate, because of the mentioned periodicity condition. This condition is valid for both the transversely bound and unbound motion, i.e., $\varepsilon_{x}<U_{0}$ and $\varepsilon_{x}>U_{0}$ (assuming the minimum of $U(x)$ is 0$)$.

The emission rate depends on the classical trajectory of the positron, which can be characterized by the momentum $\boldsymbol{p}(t)=\varepsilon \boldsymbol{v}(t)$ and the conserved particle energy

$$
\varepsilon=\sqrt{\boldsymbol{p}^{2}(t)+m^{2}}+U(x(t)) .
$$

Since in the case of channeling radiation and for our choice of the coordinates, we have that $p_{z}^{2}+p_{y}^{2}+m^{2} \gg p_{x}^{2}$ (note that $p_{y}$ and $p_{z}$ are constants of motion), and

$$
\varepsilon \approx \varepsilon_{y z}+\varepsilon_{x},
$$

where both $\varepsilon_{y z}=\sqrt{p_{z}^{2}+p_{y}^{2}+m^{2}}$ and then $\varepsilon_{x}=p_{x}^{2}(t) / 2 \varepsilon_{y z}+$ $U(x(t))$ are conserved. As mentioned, we choose the $z$ direction as the direction of the largest component of the momentum and therefore $p_{z}^{2} \gg p_{y}^{2}+m^{2}$ and $\varepsilon_{y z} \approx p_{z}+\left(p_{y}^{2}+\right.$ $\left.m^{2}\right) / 2 p_{z}$. When a photon is emitted, energy conservation implies

$$
\varepsilon_{y z, f}+\varepsilon_{x, f}+\omega=\varepsilon_{y z, i}+\varepsilon_{x, i},
$$

where the labels $i$ and $f$ denote initial and final quantities. By enforcing momentum conservation along the $y$ and the $z$ directions, we can rewrite this equation as

$$
\varepsilon_{x, f}=\varepsilon_{x, i}+\frac{p_{y, i}^{2}+m^{2}}{2 \varepsilon_{i}}-\frac{\left(p_{y, i}-k_{y}\right)^{2}+m^{2}}{2\left(\varepsilon_{i}-\omega\right)}-\omega \frac{\theta^{2}}{2},
$$

where we have used the fact that, since the photon is essentially emitted along the instantaneous positron velocity in the ultrarelativistic regime, then $\omega \approx k_{z}+\left(k_{x}^{2}+k_{y}^{2}\right) / 2 \omega \approx k_{z}+$ $(\omega \theta)^{2} / 2 \omega$. This equation indicates how to change the positron velocity accordingly after the emission of a photon takes place. This is the same formula as obtained in the quantum treatment found in Ref. [11], except that there the initial and final transverse mechanical energy $\varepsilon_{x}$ is quantized, and this decides the emission angle for a fixed value of $\omega$. Here, conversely, the semiclassical formulas give us the emission angle for a given $\omega$, and we can then obtain the change in the transverse mechanical energy in a semiclassical picture.

We therefore make the following Monte Carlo implementation for the theoretical description of the single-photon emission process: A positron starts out with initial energy $\varepsilon_{0}$ and a given initial condition $\left(\boldsymbol{r}_{\perp}(0), \boldsymbol{v}_{\perp}(0)\right)$, where the $\perp$ symbol denotes the $x$ and $y$ coordinates. Since the experiment was on planar channeling, the initial position $\boldsymbol{r}_{\perp}(0)$ has the form $\left(x_{0}, 0,0\right)$, where $x_{0}$ is a random variable uniformly distributed between 0 and the interplanar distance $d_{p}$, whereas the initial velocity $v_{\perp}(0)$ is distributed according to the experimentally measured angular distribution of the particle beam (see Table I). By choosing a time step $\Delta t$, the positron is advanced by using the Lorentz force together with the possibility that multiple scattering occurs. The size of the time step has to be such that $W \Delta t$ is much less than unity, with $W$ being the total emission rate at $t=\Delta t$. Moreover, $\Delta t$ has to correspond to a length much smaller than the dechanneling length, which is the length over which the multiple scattering 
would, on average, have increased the transverse positron energy by the total potential depth $U_{0}\left[U_{0}\right.$ is equal to $22.7 \mathrm{eV}$ in the case of the silicon (110) plane], implying in turn that the particle would no longer be bound. The rate $W$ is evaluated by integrating the differential rate $d^{2} W / d \omega d \Omega$ in Eq. (A1), with a solution of the Lorentz-force equation with initial conditions given by the positron position and velocity at $t=\Delta t$ and for a duration $T$ from $t=\Delta t-T / 2$ to $t=\Delta t+T / 2$. The time interval $T$ is chosen such that the limit convergence of the integral in Eq. (A1) is achieved. As mentioned, this is possible by choosing $T \gg \max \left(l_{f}, \lambda\right)$. Indeed, the formation length of a photon with energy $\omega$ is given by $l_{f}=2 \gamma^{2}(1-\omega / \varepsilon) / \omega$ [3], with $\gamma=\varepsilon / m$ being the Lorentz factor of the positron at the moment of emission. For $\omega \approx \chi \varepsilon$, it is $l_{f} \sim 2 \gamma \lambda_{C} / \chi$, where $\lambda_{C}=1 / m=3.9 \times 10^{-11} \mathrm{~cm}$ is the Compton wavelength, and, for the parameters of the experiment, $l_{f} \sim 1 \mu \mathrm{m}$. The time interval $T$ has been chosen to be multiple times the formation length (divided by the speed of light), or the wavelength of the motion, whichever is larger, by checking numerically that the mentioned convergence was achieved. Moreover, as the formation length is typically $\alpha \approx 1 / 137$ times smaller than the radiation length, for which the emission probability is unity, $\Delta t$ can be chosen to be larger than $T$ (notice that under the assumption of being in the channeling regime, the dechanneling length is much longer than the formation length too). Now, since $\Delta t$ is such that $W \Delta t \ll 1$, a random number is sampled to decide whether the radiation emission takes place. If radiation emission takes place, the value of $\omega$ is decided based on the calculated distribution $d W / d \omega$ and the inverse transform sampling method. Once $\omega$ has been picked, the angle of emission is picked using the distribution $d^{2} W / d \omega d \Omega$ and a Monte Carlo acceptancerejection method. The emission is assumed to occur at $t=\Delta t$ and the new value of the positron energy is $\varepsilon_{0}-\omega$, whereas the new value of the transverse mechanical energy has to be consistent with Eq. (A7). This process is then repeated for all time steps $\Delta t$ until the end of the crystal is reached.

We point out that, in principle, multiple solutions for the new initial conditions in $x$ and $v_{x}$ are possible if the photon emission occurs. However, we have made sure numerically that, provided that the transverse mechanical energy is given

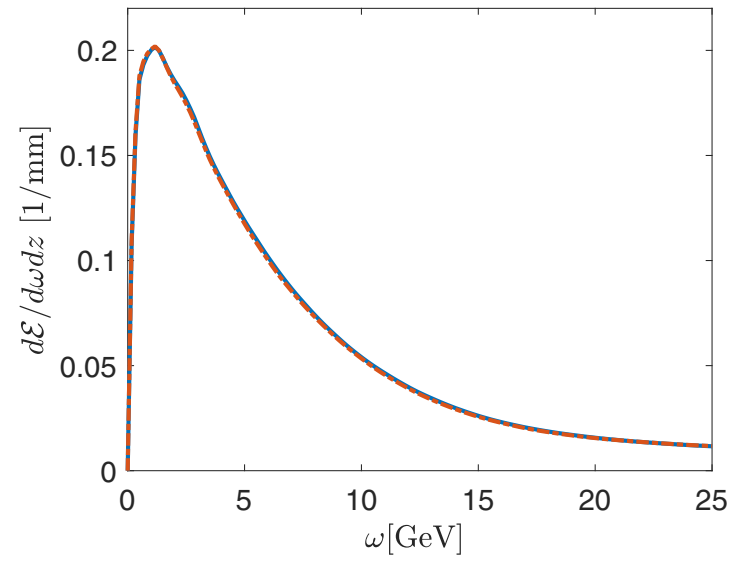

FIG. 4. Two photon spectra for the parameters $L=6.2 \mathrm{~mm}$ and $\sigma_{\theta}=85 \mu \mathrm{rad}$. The blue continuous curve (red dashed curve) corresponds to selecting the initial position of the positron along the $x$ direction to be centered between two planes [half the maximal value allowed from Eq. (A7)]. For each value, the initial velocity along the $x$ direction has been chosen in such a way that the initial transverse mechanical energy of the positron is the same (both possible values of $v_{x}$ differing only by the sign provide indistinguishable results).

by Eq. (A7), all different initial conditions provide essentially the same photon spectra. The physical reason of this is that the emission probability in a crystal for planar channeling depends on the transverse mechanical energy (as well as on the total energy) of the emitting particle but not on the precise initial values of $x$ and $v_{x}$. For the sake of completeness, we show in Fig. 4 the results of two calculations for the parameters $L=6.2 \mathrm{~mm}$ and $\sigma_{\theta}=85 \mu \mathrm{rad}$. The two curves correspond to choosing the initial $x$ position of the positron at each emission to be either centered between two planes (blue continuous curve) or to be half the maximal value allowed from Eq. (A7) (red dashed curve). Moreover, we have ensured that also for the initial positron $x$ coordinate, being the maximum value allowed by Eq. (A7), the results are unchanged. This conclusion was also verified in all cases for both possible values of the initial velocity $v_{x}$, differing only by the sign.
[1] H. Mitter, Acta Phys. Austriaca XIV, 397 (1975).

[2] V. I. Ritus, J. Sov. Laser Res. 6, 497 (1985).

[3] V. Baier, V. Katkov, and V. Strakhovenko, Electromagnetic Processes at High Energies in Oriented Single Crystals (World Scientific, Singapore, 1998).

[4] A. Di Piazza, C. Müller, K. Z. Hatsagortsyan, and C. H. Keitel, Rev. Mod. Phys. 84, 1177 (2012).

[5] U. I. Uggerhøj, Rev. Mod. Phys. 77, 1131 (2005).

[6] M. Fuchs, M. Trigo, J. Chen, S. Ghimire, S. Shwartz, M. Kozina, M. Jiang, T. Henighan, C. Bray, G. Ndabashimiye, P. H. Bucksbaum, Y. Feng, S. Herrmann, G. A. Carini, J. Pines, P. Hart, C. Kenney, S. Guillet, S. Boutet, G. J. Williams, M. Messerschmidt, M. M. Seibert, S. Moeller, J. B. Hastings, and D. A. Reis, Nat. Phys. 11, 964 (2015).
[7] V. B. Berestetskii, E. M. Lifshitz, and L. P. Pitaevskii, Quantum Electrodynamics (Elsevier Butterworth-Heinemann, Oxford, UK, 1982).

[8] J. Lindhard, K. Dan. Vidensk. Selsk. Mat. Fys. Medd. 341 (1965).

[9] J. U. Andersen, phys.au.dk/publikationer/lecture-notes

[10] A. I. Akhiezer and N. F. Shul'ga, High-Energy Electrodynamics in Matter (Gordon and Breach, Amsterdam, 1996).

[11] T. N. Wistisen and A. Di Piazza, Phys. Rev. D 99, 116010 (2019).

[12] V. Baier, V. Katkov, and V. Strakhovenko, Usp. Fiz. Nauk. 159, 455 (1989).

[13] T. N. Wistisen, Phys. Rev. D 100, 036002 (2019).

[14] R. Blankenbecler and S. D. Drell, Phys. Rev. D 53, 6265 (1996). 
[15] T. N. Wistisen, Phys. Rev. D 92, 045045 (2015).

[16] A. Di Piazza, M. Tamburini, S. Meuren, and C. H. Keitel, Phys. Rev. A 98, 012134 (2018).

[17] T. G. Blackburn, D. Seipt, S. S. Bulanov, and M. Marklund, Phys. Plasmas 25, 083108 (2018).

[18] A. Di Piazza, M. Tamburini, S. Meuren, and C. H. Keitel, Phys. Rev. A 99, 022125 (2019).

[19] A. Ilderton, B. King, and D. Seipt, Phys. Rev. A 99, 042121 (2019).

[20] I. A. Aleksandrov, G. Plunien, and V. M. Shabaev, Phys. Rev. D 99, 016020 (2019).

[21] V. N. Baier, V. M. Katkov, and V. M. Strakhovenko, Nucl. Phys. B 328, 387 (1989).

[22] M. K. Khokonov and H. Nitta, Phys. Rev. Lett. 89, 094801 (2002).

[23] A. Belkacem, G. Bologna, M. Chevallier, N. Cue, M. Gaillard, R. Genre, J. Kimball, R. Kirsch, B. Marsh, J. Peigneux, J. Poizat, J. Remillieux, D. Sillou, M. Spighel, and C. Sun, Phys. Lett. B 177, 211 (1986).

[24] J. Bak, J. Ellison, B. Marsh, F. Meyer, O. Pedersen, J. Petersen, E. Uggerh $\varnothing$ j, K. Østergaard, S. Møller, A. Sørensen, and M. Suffert, Nucl. Phys. B. 254, 491 (1985).

[25] L. Bandiera, E. Bagli, V. Guidi, A. Mazzolari, A. Berra, D. Lietti, M. Prest, E. Vallazza, D. De Salvador, and V. Tikhomirov, Phys. Rev. Lett. 111, 255502 (2013).

[26] M. K. Khokonov, Phys. Lett. B 791, 281 (2019).

[27] N. Shul'ga and S. Fomin, JETP Lett. 27, 117 (1978).

[28] S. Fomin and N. Shul'ga, Phys. Lett. A 73, 131 (1979).
[29] T. N. Wistisen, A. Di Piazza, H. V. Knudsen, and U. I. Uggerhøj, Nat. Commun. 9, 795 (2018).

[30] T. N. Wistisen and A. Di Piazza, Phys. Rev. A 98, 022131 (2018).

[31] A. Di Piazza, K. Z. Hatsagortsyan, and C. H. Keitel, Phys. Rev. Lett. 105, 220403 (2010).

[32] J. M. Cole, K. T. Behm, E. Gerstmayr, T. G. Blackburn, J. C. Wood, C. D. Baird, M. J. Duff, C. Harvey, A. Ilderton, A. S. Joglekar, K. Krushelnick, S. Kuschel, M. Marklund, P. McKenna, C. D. Murphy, K. Poder, C. P. Ridgers, G. M. Samarin, G. Sarri, D. R. Symes, A. G. R. Thomas, J. Warwick, M. Zepf, Z. Najmudin, and S. P. D. Mangles, Phys. Rev. X 8, 011020 (2018).

[33] K. Poder, M. Tamburini, G. Sarri, A. Di Piazza, S. Kuschel, C. D. Baird, K. Behm, S. Bohlen, J. M. Cole, D. J. Corvan, M. Duff, E. Gerstmayr, C. H. Keitel, K. Krushelnick, S. P. D. Mangles, P. McKenna, C. D. Murphy, Z. Najmudin, C. P. Ridgers, G. M. Samarin, D. R. Symes, A. G. R. Thomas, J. Warwick, and M. Zepf, Phys. Rev. X 8, 031004 (2018).

[34] V. Baier and V. Katkov, J. Exp. Theor. Phys. 26, 854 (1968).

[35] A. Belkacem, N. Cue, and J. Kimball, Phys. Lett. A 111, 86 (1985).

[36] J. Kimball, N. Cue, and A. Belkacem, Nucl. Inst. Meth. B 13, 1 (1986).

[37] T. N. Wistisen, Phys. Rev. D 90, 125008 (2014).

[38] A. Babaev and S. B. Dabagov, Eur. Phys. J. Plus 127, 62 (2012).

[39] X. Artru, Nucl. Instrum. Meth. B 48, 278 (1990). 